\title{
Coverage-dependent formic acid oxidation reaction kinetics determined by oscillating potentials
}

\author{
Max J. Hülsey ${ }^{1, \#}$, Chia Wei Lim ${ }^{1, \#}$, Sie Shing Wong ${ }^{1,2}$, and Ning Yan ${ }^{1 *}$ \\ ${ }^{1}$ National University of Singapore, Department of Chemical and Biomolecular Engineering, 4 Engineering Drive \\ 4, 117585 Singapore. \\ ${ }^{2}$ Joint School of National University of Singapore and Tianjin University, International Campus of Tianjin \\ University, Binhai New City, Fuzhou 350207, China. \\ \# Authors contributed equally. \\ * Corresponding author: ning.yan@ nus.edu.sg
}

\begin{abstract}
Establishing correlations between catalytic activity and dynamic surface properties of real catalysts, such as adsorbate coverage, is not straight-forward but crucial for the understanding of catalytic phenomena. The formic acid oxidation reaction comprises the non-Faradaic dehydration to $\mathrm{CO}$ when no potential is applied to the catalyst surface and the subsequent Faradaic oxidative desorption to form $\mathrm{CO}_{2}$. Here, we report a methodology based on applying oscillating potentials to various electrocatalytically active metal surfaces during the formic acid oxidation reaction. Moderate frequency oscillations $(0.1$ to $10 \mathrm{~Hz})$ allow us to control the coverage of intermediates on the surface, thus enable quantifying the transient effects (on the time scale of up to $10^{-4} \mathrm{~s}$ ) of coverage on the reaction rate. We determined different coverage-dependences of turnover frequencies for Pt metal plate and various carbonsupported metal nanoparticle catalysts $(\mathrm{Pt} / \mathrm{C}, \mathrm{Pd} / \mathrm{C}$ and $\mathrm{Rh} / \mathrm{C})$. This method therefore constitutes a valuable and simple tool for the elucidation of adsorbate coverages on metal surfaces and their resulting catalytic performance. We also demonstrate that dynamic catalytic processes can be analyzed semi-quantitatively with this new approach allowing the design of catalytic processes under optimized conditions.
\end{abstract}

\section{Introduction}

Dynamic effects of electric potentials on catalytic surfaces have been vastly ignored so far despite the wide-spread use of electroanalytic techniques like electrochemical impedance spectroscopy which relies on high-frequency potential changes. One of the earliest reports by Adžić et al. showed formic acid (FA) electrooxidation enhancements when 'pulsated potentials' with an amplitude of $600 \mathrm{mV}$ and an ideal frequency of $2000 \mathrm{~Hz}$ were applied to a Pt electrode. Current density improvements on the order of $10^{2}$ were achieved. ${ }^{1}$ Similar trends were established for the methanol oxidation reaction on Pt surfaces again with current density increases of around $10^{2} .{ }^{2}$ Catalyst morphology changes under oscillating potentials were predicted by Kim et al. ${ }^{3}$ who proposed that this may explain experimental catalytic results obtained earlier on $\mathrm{Pd} / \mathrm{LiNbO}_{3}$ catalysts. ${ }^{4}$ Kakekhani et al. conducted systematic first-principle calculations on the effect of electric polarization switches for the reaction of $\mathrm{NO}$ and $\mathrm{CO}$ over $\mathrm{RuO}_{2} / \mathrm{PbTiO}_{3}$ and $\mathrm{CrO}_{2} / \mathrm{PbTiO}_{3}$. In line with previous simulations, they found that polarization could change the oxidation state of surface metals by up to \pm 1 . Thus, depending on the polarization of the substrate, binding energies of adsorbates could be modified to different extents thus affecting surface coverages and reactivities. In this 'cyclic catalysis' manner, the authors proposed that the well-known Sabatier limit could be overcome. ${ }^{5}$ In fact, the variation of adsorption energies by applied potentials and thus impacted catalytic performance has been investigated experimentally and theoretically before. ${ }^{6-7}$ For oscillating potentials, enhancement effects have also been reported for the electrochemical adiponitrile synthesis. $^{8} \quad$ Conventionally, the adiponitrile electrosynthesis suffers from undesired side reactions due to the concomitant accumulation of product and drop in substrate concentration in proximity to the electrode surface. Switching the surface polarization between two states with a square waveform enabled the fast reaction of substrate (cathodic time) as well as the diffusion of product from and substrate towards the electrode (anodic resting time). Beyond this, Blanco et al. claimed that electrolyte cations could diffuse towards the electrode during the anodic resting time, 
which was reported to enhance the adiponitrile selectivity. Machine-learning aided optimization of various reaction parameters enabled improvements in the adiponitrile production rates and selectivities of $30 \%$ and $325 \%$, respectively. ${ }^{8}$ Based on insightful microkinetic modeling on the effects of dynamic switching of surface properties, Ardagh et al. revealed that both the reactivity as well as the selectivity of competing reactions can be modified dramatically if the changes in (relative) adsorption energies are sufficiently high. It was further predicted that oscillations need to be in approximate resonance with the surface reactions to achieve reaction enhancements. ${ }^{9-12}$ DFT calculations by Shetty et al. revealed electric field-dependent linear scaling relationships of adsorbates on metal surfaces imperative for the understanding of dynamic catalytic processes. ${ }^{13}$ Cycling between a potential suitable for the non-Faradaic dehydration of FA to surfaceadsorbed $\mathrm{CO}$ and the Faradaic oxidative desorption to form $\mathrm{CO}_{2}$ enhanced the activity by a factor of up to around 20 at frequencies of $100 \mathrm{~Hz},{ }^{14}$ consistent with promotional effect observed by Adžić et al. earlier. ${ }^{1}$

Besides enhancing surface reactions, we envisage that oscillating potentials are capable of aiding the investigation of transient surface properties during catalysis. Transient effects are known to impact electrocatalytic reaction rates tremendously. One of those effects is the coverage of the surface with adsorbates affecting catalysis in diverse ways. For many multimolecular surface reactions, balanced relative adsorption strengths of all reactants are required for efficient surface reactions. Furthermore, lateral interactions between adsorbates as well as electronic effects that adsorbates have on metal atoms in close proximity play pivotal roles. Unfortunately, tools to study coverages and rates simultaneously on real catalysts in liquid phase reactions are rare and suffer from serious shortcomings. Some techniques (e.g., X-ray photoelectron spectroscopy and in situ electron microscopy) are not applicable to study reactions in solvents, while some others provide information on bulk solid materials (e.g., X-ray absorption spectroscopy, Mößbauer spectroscopy, Xway diffraction) thus offering limited insights into the surface chemistry. One of the most developed techniques to analyze surface chemistry during electrocatalysis is vibrational spectroscopy (IR/Raman) but the time resolution is often not sufficient to capture transient surface effects and it is not selective to electrocatalytic reactions. ${ }^{15}$ Surface coverage with intermediates can also be influenced by poisoning the electrocatalytically active surface area with more inert metals although conclusions are also affected by bimetallic alloying effects. ${ }^{16-17}$ Due to the inability of experimental approaches to investigate lateral adsorbate interactions, most available information stems from DFT calculations. For example, atomistic simulations reveal that, for the $\mathrm{CO}$ oxidation reaction, adsorbate-adsorbate interactions do not affect which metal is the most catalytically active but are capable of asymmetrically broadening volcano curves. This has been shown to be dependent on the type of the metal but those trends are not easily confirmed experimentally. ${ }^{18}$

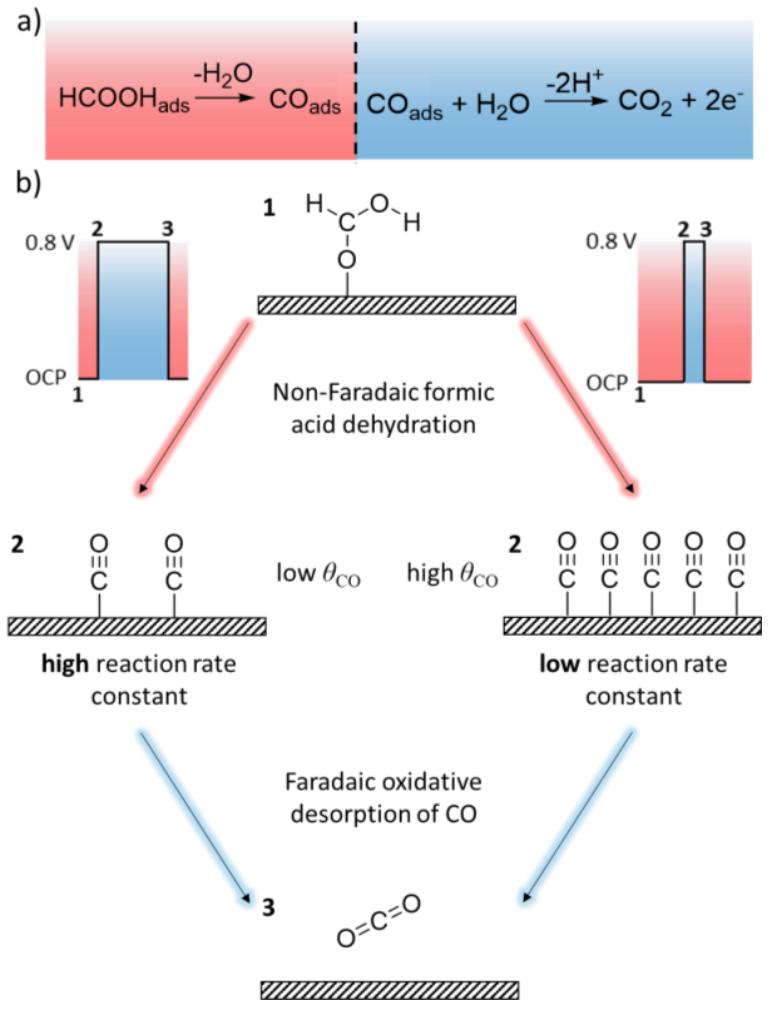

Figure 1. Overview of our electrochemical approach based on oscillating potentials. a) Non-Faradaic and Faradaic steps of the FA oxidation reaction; b) schematic of our approach for determining the coverage-dependent reaction rate constant.

Herein, we describe the development of a simple technique relying on the application of oscillating potentials between the open-circuit potential (OCP) and $0.8 \mathrm{~V}_{\mathrm{NHE}}$ (potential against the normal hydrogen electrode) on different catalyst surfaces during the formic acid (FA) oxidation reaction. As reported previously, the open-circuit potential favors the nonFaradaic FA dehydration resulting in a metal surface covered with $\mathrm{CO}$ (Figure 1a). The oxidative desorption of $\mathrm{CO}$ only proceeds at higher potentials with the onset 
around $0.8 \mathrm{~V}_{\text {NHE. }}$ Therefore, cycling between those two potentials enables controlled coverage of the surface with $\mathrm{CO}$ adsorbates (illustrated in red in Figure 1b) and the measurement of Faradaic reaction rate by detecting the current density (illustrated in blue in Figure 1b). We demonstrated that this approach allows us to determine $\mathrm{CO}$ coverage-dependent reaction rates on different metal surfaces.

\section{Materials and Methods}

\subsection{Catalyst preparation and characterization}

Carbon cloths (Xinke Experimental Materials Sales Center) were first pre-treated by immersing in $32.5 \%$ nitric acid, made from nitric acid (VWR Chemicals, $65 \%$ ) and ultra-pure water (Milli-Q®), and heating at $80{ }^{\circ} \mathrm{C}$ for $24 \mathrm{~h}$. The carbon cloths were then thoroughly rinsed with ultra-pure water and dried overnight. To prepare $\mathrm{Pt} / \mathrm{C}$ catalyst ink, $2.1 \mathrm{mg}$ of $5 \mathrm{wt} \% \mathrm{Pt} / \mathrm{C}$ (Sigma-Aldrich), $0.5 \mathrm{mg}$ of Nafion ${ }^{\circledR}$ perfluorinated resin solution (Sigma-Aldrich, $5 \mathrm{wt} \%$ in $45 \%$ water balanced with lower aliphatic alcohols), $0.6 \mathrm{mg}$ of finely ground activated charcoal (Sigma-Aldrich, 12-20 mesh granular DARCO®) and $700 \mu \mathrm{L}$ of absolute ethanol (VWR chemicals, >99.7\%) were mixed and sonicated for $1.5 \mathrm{~h}$. For Pd/C catalyst ink, $1.1 \mathrm{mg}$ of 10 wt\% Pd/C (Sigma-Aldrich) and $1.6 \mathrm{mg}$ of activated charcoal were used, while for $\mathrm{Rh} / \mathrm{C}$ catalyst ink, $2.1 \mathrm{mg}$ of $5 \mathrm{wt} \% \mathrm{Rh} / \mathrm{C}$ (Shaanxi Kaida Chemical Engineering Co. Ltd.) and $0.6 \mathrm{mg}$ of activated charcoal were used. The prepared catalyst ink was transferred to both sides of a $1 \times 1 \mathrm{~cm}$ pre-treated carbon cloth. The impregnated carbon cloths were dried at $80^{\circ} \mathrm{C}$ overnight and were then rinsed with and stored in ultra-pure water until use.

The JEM 2100F (JEOL, Japan) microscope was operated at $200 \mathrm{kV}$ to collect transmission electron microscopy (TEM) images. A Bruker D8 Advance Xray diffractometer was used to collect $\mathrm{X}$-ray diffraction pattern of different commercial carbon-supported catalyst materials between 5 and $80^{\circ}$. The dispersion of the Pt plate electrode was estimated based on simple geometric arguments and the density of atoms per surface area for flat $\mathrm{Pt}$ surfaces $\left(10^{19} \mathrm{~m}^{-2}\right)$. The dispersion of carbon-based catalysts was approximated based on the following empirical correlation which was shown to be valid for dispersions between around 20 and $92 \%$ :

$$
D=\sqrt[1.23]{3.32 \times \frac{d_{\mathrm{at}}}{d_{\mathrm{AV}}}}
$$

With $D$ as dispersion, $d_{\text {at }}$ as the atomic radius $(200 \mathrm{pm}$ for $\mathrm{Rh}, 163 \mathrm{pm}$ for $\mathrm{Pd}$, and $175 \mathrm{pm}$ for $\mathrm{Pt}$ ) and $d_{\mathrm{AV}}$ for the average particle size. ${ }^{19}$

\subsection{Electrochemical setup and experiments}

For all the experiments, a $200 \mathrm{~cm}^{3}$ three-electrode electrochemical cell was used, with a Pt plate electrode (Shanghai Jingchong Electronic Technology Development Pte. Ltd.) as the counter electrode and a saturated calomel electrode (Shanghai Jingchong Electronic Technology Development Pte. Ltd.) as the reference electrode. The working electrode was either a Pt plate electrode or one of the impregnated carbon cloths prepared earlier. $150 \mathrm{~cm}^{3}$ aqueous solution of 0.25 M FA (Sigma-Aldrich, >95\%) and 0.25 M sulfuric acid (Sigma-Aldrich, 99.999\%) was used as the electrolyte. Purified nitrogen gas (Air Liquide, $99.9995 \%$ ) was continuously passed through the

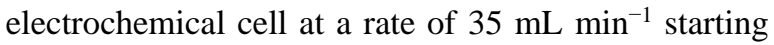
from $1 \mathrm{~h}$ before experiments. The electrolyte solution was stirred at a constant speed of $800 \mathrm{rpm}$ and all experiments were conducted at room temperature.

A Gamry Reference 3000 Potentiostat was used for all electrochemical measurements. To obtain the open circuit potential (OCP) of the electrochemical system, the measurement was conducted until the OCP reading stabilized within a tolerance of $10 \mathrm{mV}$ for $500 \mathrm{~s}$ (Figure S1). For Cyclic Voltammetry (CV) measurements, a scan rate of $50 \mathrm{mV} \mathrm{s}^{-1}$ and a step size of $5 \mathrm{mV}$ were used for Pt plate while a scan rate of $10 \mathrm{mV} \mathrm{s}^{-1}$ and a step size of $5 \mathrm{mV}$ were used for the carbon-supported metal catalysts due to the very high current observed at higher scan rates. CV measurements were repeated until there was no further change between successive CV curves. For Electrochemical Impedance Spectroscopy (EIS) measurements, the frequency ranged from 1000 to $0.1 \mathrm{~Hz}$ with an AC voltage of $10 \mathrm{mV} \mathrm{rms}$. Using the Gamry Virtual Front Panel software, oscillating electric potentials of square waveforms were applied to the working electrode at a data acquisition frequency of $5000 \mathrm{~Hz}$. The maximum of the square waves was 0.8 $\mathrm{V}_{\mathrm{NHE}}$ while the minimum was the OCP value measured earlier. The wave frequency ranged from 0.1 to $10 \mathrm{~Hz}$ while the duty cycle was varied between 0.01 and 99.995\%. Each experiment involving oscillating potentials lasted $100 \mathrm{~s}$ and the data for last complete cycle was extracted for analysis. In this paper, all potential values have been reported with reference to the normal hydrogen electrode (NHE). $\mathrm{CO}_{2}$ was separated and quantified with an Agilent 7890B gas 
chromatograph with a TCD detector. At each static or dynamic potential, gas samples were taken continuously until three subsequent measurements showed deviations in $\mathrm{CO}_{2}$ formation rates of below $1 \%$. Calibrations were done with a $1 \% \mathrm{CO}_{2}$ (Air Liquide, balance $\mathrm{N}_{2}$ ) gas mixture. Frequency $(f)$ and duty cycle are defined as follows:

$$
\begin{aligned}
& f=\frac{1}{t_{\mathrm{OCP}}+t_{0.8 \mathrm{~V}}} \\
& \text { duty cycle }=\frac{t_{0.8 \mathrm{~V}}}{t_{\mathrm{OCP}}+t_{0.8 \mathrm{~V}}} \times 100 \%
\end{aligned}
$$

\section{Results and Discussion}

\subsection{Catalyst characterization}

Pt plate electrodes were purchased and used as received while commercial $\mathrm{Pt} / \mathrm{Pd} / \mathrm{Rh}$ nanoparticles supported on carbon were prepared by drop-casting their ethanolbased inks on carbon cloth. TEM images were used to determine the average particle size for the commercial carbon-based catalysts. $1.95 \pm 0.34 \mathrm{~nm}, 2.82 \pm 0.41 \mathrm{~nm}$, and $5.4 \pm 0.95 \mathrm{~nm}$ were identified for $\mathrm{Pt}, \mathrm{Pd}$, and $\mathrm{Rh} / \mathrm{C}$, respectively (Figure S2). XRD patterns for each of the materials show no clear diffraction peaks for these metals confirming the size of metal particles to be small (Figure S3). Using equation (1), dispersions were estimated to be $26 \%$ for $\mathrm{Pd} / \mathrm{C}, 37 \%$ for $\mathrm{Pt} / \mathrm{C}$, and $18 \%$ for $\mathrm{Rh} / \mathrm{C}$. Those values are consistent with previous studies on comparable carbon-supported catalysts. ${ }^{20}$ Therefore, we calculated the turnover frequencies on carbon-based catalysts based on the dispersion values while the number of sites of the Pt plate electrode was calculated based on the total surface area and the approximate Pt site density.

CV scans with sulfuric acid/FA electrolytes on Pt plate electrodes revealed two redox features during the anodic trace (Figure 2). The first one centered at around $0.5 \mathrm{~V}$ is commonly associated to Faradaic FA oxidation reactions to surface formate or hydroxy carbonyl intermediates while the second one occurs due to the Faradaic $\mathrm{CO}$ oxidative desorption reaction. For carbon-based electrodes, the CV peaks are less easily discernable but similar peak shapes to Pt plates have been reported before. ${ }^{21-24}$

The effect of oscillating potentials on the FA oxidation reaction on $\mathrm{Pt}$ plate electrodes has been investigated and we identified a frequency of $100 \mathrm{~Hz}$ at a duty cycle of $50 \%$ between OCP and $0.8 \mathrm{~V}$ to yield the largest FA oxidation enhancement (Figure S4) in accordance to a previous study. ${ }^{14}$ In the following, we further elucidated the transient current responses upon potential switches and we explored the capability of oscillating potentials in illuminating reaction kinetics.

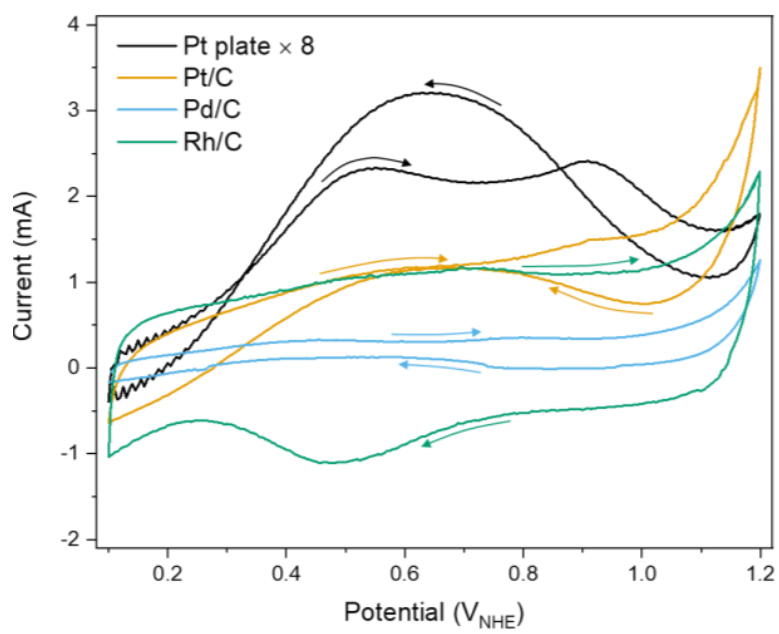

Figure 2. CV scans of different catalysts in $0.25 \mathrm{M}$ $\mathrm{H}_{2} \mathrm{SO}_{4}$ and $0.25 \mathrm{M}$ FA. Scan rates were $10 \mathrm{mV} \mathrm{s}^{-1}$ (for Pt plate: $50 \mathrm{mV} \mathrm{s}^{-1}$ ).

\subsection{Modelling of transient current responses}

Applying oscillating potentials between the OCP and $0.8 \mathrm{~V}$ on electrodes in sulfuric acid/FA electrolyte gave transient current responses which should arise mostly due to the Faradaic oxidation of $\mathrm{CO}$ on the electrode surface. As shown in Figure 3, the current decays in an exponential-like manner over time.

At OCP, the dehydration of FA takes place and the produced $\mathrm{CO}$ is adsorbed on the metal catalyst surface. When the potential changes from OCP to $0.8 \mathrm{~V}$, the adsorbed $\mathrm{CO}$ starts to undergo Faradaic oxidative desorption, resulting in a current flow. Simultaneously, the adsorption and dehydration of FA is severely prohibited at this high potential as shown earlier. ${ }^{25}$ The turnover frequency (TOF) for oxidative desorption was modelled as:

$$
\mathrm{TOF}=k_{1} \theta
$$

where $k_{1}$ is the reaction rate constant and $\theta$ represents $\mathrm{CO}$ coverage on the catalyst surface.

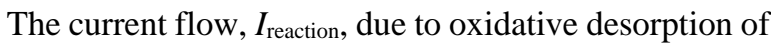
$\mathrm{CO}$ was given by:

$$
I_{\text {reaction }}=\mathrm{TOF} \times N_{\text {sites }} \times m \times F
$$

where $N_{\text {sites }}$ is the number of moles of active sites on the catalyst surface, $m(=2)$ is the number of electrons given out for each $\mathrm{CO}$ oxidized and $F$ $\left(=96500 \mathrm{C} \mathrm{mol}^{-1}\right)$ is the Faraday constant. 
Since TOF $=-\frac{d \theta}{d t}$, integration of (4) gave

$$
\theta=\theta_{\mathrm{i}} \exp \left(-k_{1} t\right)
$$

where $\theta_{\mathrm{i}}$ is the CO coverage at $t=0$.

Combining (5) and (6), the current can be represented as:

$$
\begin{aligned}
& I_{\text {reaction }}=N_{\text {sites }} \times m \times F \times k_{1} \times \\
& \theta_{\mathrm{i}} \exp \left(-k_{1} t\right)
\end{aligned}
$$

We found that the transient current response of our system cannot be modelled sufficiently well by a single exponential term, suggesting that the current response comprises a more complex physical behavior than initially assumed (vide infra). This led us to the assumption that the impedance response of electrochemical systems under rapidly oscillating potentials might be non-negligible. Any electrochemical system gives current responses whenever the applied potential is changed, even in the absence of any reaction, due to its inherent impedance. Electrochemical impedance spectra were fitted with the system modelled as a Randles circuit, which consists of two resistors (bulk electrolyte resistance, $R_{1}$ and charge transfer resistance, $R_{2}$ ) and a capacitor (interfacial double-layer capacitance, $C$ ). $R_{2}$ is in parallel with $C$, while both of them are in series with $R_{1}$ (Figure S5). ${ }^{26-}$ ${ }^{27}$ We used $V_{\mathrm{A}}$ and $V_{\mathrm{B}}$ to denote the potential differences across $R_{1}$ and the parallel branch of $R_{2}$ and $\mathrm{C}$ respectively. Letting $I_{\mathrm{A}}$ and $I_{\mathrm{B}}$ be the currents through $R_{2}$ and $C$ respectively, the current response, $I_{\text {background }}(t)$, of a Randles circuit following a step increase in applied potential from open circuit to $V$ at $t=0$ was derived:

$$
\begin{aligned}
& V_{\mathrm{A}}=\left(I_{\mathrm{A}}+I_{\mathrm{B}}\right) R_{1} \\
& V_{\mathrm{B}}=I_{\mathrm{A}} R_{2}=\frac{1}{C} \int I_{\mathrm{B}} d t \\
& V=V_{\mathrm{A}}+V_{\mathrm{B}}=\left(I_{\mathrm{A}}+I_{\mathrm{B}}\right) R_{1}+I_{\mathrm{A}} R_{2} \\
& \Rightarrow I_{\mathrm{A}}=\frac{V-I_{\mathrm{B}} R_{1}}{R_{1}+R_{2}}
\end{aligned}
$$

Alternatively, we can also write

$$
V=V_{\mathrm{A}}+V_{\mathrm{B}}=\left(I_{\mathrm{A}}+I_{\mathrm{B}}\right) R_{1}+\frac{1}{C} \int I_{\mathrm{B}} d t
$$

Differentiating (11) with respect to $t$,

$$
\frac{d V}{d t}=\left(\frac{d I_{\mathrm{A}}}{d t}+\frac{d I_{\mathrm{B}}}{d t}\right) R_{1}+\frac{1}{C} I_{\mathrm{B}}
$$

Since $\frac{d V}{d t}=0$ when $t>0$ for a step change, combining (10) and (12) gave

$$
\begin{aligned}
& {\left[\frac{1}{R_{1}+R_{2}}\left(\frac{d V}{d t}-R_{1} \frac{d I_{\mathrm{B}}}{d t}\right)+\frac{d I_{\mathrm{B}}}{d t}\right] R_{1}+\frac{1}{C} I_{\mathrm{B}}=} \\
& 0
\end{aligned}
$$

Using again $\frac{d V}{d t}=0$ and after some rearrangement,

$$
\frac{d I_{\mathrm{B}}}{d t}=-\frac{1}{C} \frac{R_{1}+R_{2}}{R_{1} R_{2}} I_{\mathrm{B}}
$$

Integration of (14) gave

$$
I_{\mathrm{B}}=I_{\mathrm{Bi}} \exp \left(-\frac{1}{C} \frac{R_{1}+R_{2}}{R_{1} R_{2}} t\right)
$$

where $I_{\mathrm{Bi}}$ is the current through the capacitor at $t=0^{+}$.

Now, using (10),

$$
\begin{aligned}
& I=I_{\mathrm{A}}+I_{\mathrm{B}} \\
& =\frac{1}{R_{1}+R_{2}}\left[V-R_{1} I_{\mathrm{Bi}} \exp \left(-\frac{1}{C} \frac{R_{1}+R_{2}}{R_{1} R_{2}} t\right)\right] \\
& +I_{\mathrm{Bi}} \exp \left(-\frac{1}{C} \frac{R_{1}+R_{2}}{R_{1} R_{2}} t\right)
\end{aligned}
$$

Simplifying gave

$$
\begin{aligned}
& I_{\text {background }}(t) \\
& =\frac{V}{R_{1}+R_{2}}+\frac{R_{2}}{R_{1}+R_{2}} I_{\mathrm{Bi}} \exp \left(-\frac{1}{C} \frac{R_{1}+R_{2}}{R_{1} R_{2}} t\right)
\end{aligned}
$$

The values of resistances and capacitance were obtained from fitting the EIS results in a dilute sulfuric acid electrolyte using the Randles circuit model (Figure S5): $R_{1}=2.525 \Omega, R_{2}=9.519 \times 10^{3} \Omega, C=$ $7.06 \times 10^{-4} \mathrm{~F}$. Since $R_{2}$ was much greater than $R_{1}$, the expression for $I_{\text {background }}(t)$ can be simplified to give

$$
I_{\text {background }}(t)=I_{\mathrm{Bi}} \exp \left(-\frac{t}{R_{1} C}\right)
$$

Physically, this implies that the current $I_{\mathrm{A}}$ through resistor $R_{2}$ is much smaller compared to the current through the capacitor, $I_{\mathrm{B}}$. This is because the resistance, $R_{2}$, was much larger in magnitude than the capacitor impedance, $C$. The electrochemical system effectively behaved like a simple RC series circuit with an exponentially decaying current response when subject to a step change in applied potential from an initial state of open circuit.

From above, the current measured during the high potential cycle $(0.8 \mathrm{~V})$ of the electrochemical experiments is the sum of the two contributions: (1) 
current due to electron transfer for the oxidative desorption of $\mathrm{CO}$, and (2) background current response due to impedance of the electrochemical system. The following equation was used to model the current measured, $I_{\text {total }}$, to obtain the relevant parameters through minimizing the residual sum of squares (RSS):

$$
\begin{aligned}
& I_{\text {total }}=I_{\text {reaction }}+I_{\text {background }} \\
& =A_{1} \exp \left(-k_{1} t\right)+A_{2} \exp \left(-k_{2} t\right)
\end{aligned}
$$

where $A_{1}=N_{\text {sites }} \times m \times F \times k_{1} \times \theta_{\mathrm{i}}, A_{2}=I_{\mathrm{Bi}}$ and $k_{2}=\frac{1}{R_{1} C}$.

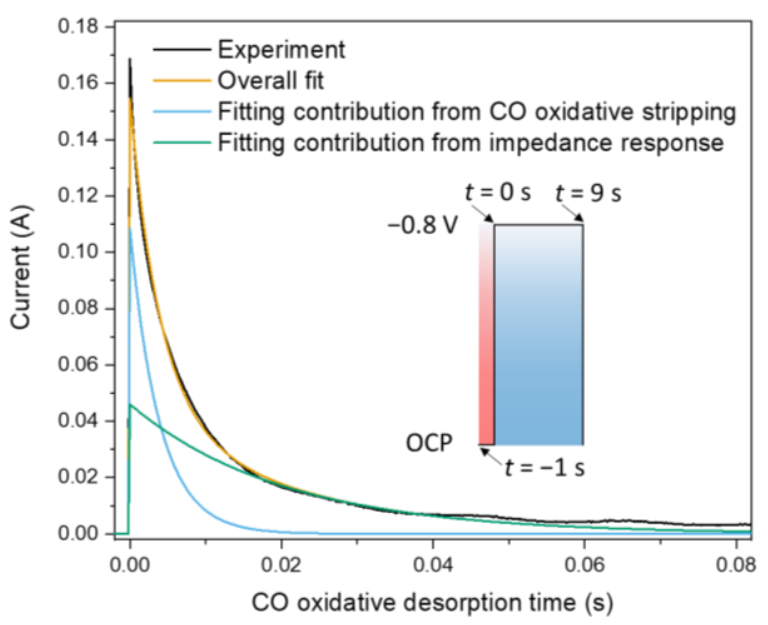

Figure 3. Transient current response during square wave shaped potential oscillations at $0.1 \mathrm{~Hz}(90 \%$ duty cycle). Different contributions to the experimentally observed current response were plotted alongside the overall fit. The plotted current response was directly after switching from OCP to $0.8 \mathrm{~V}$. The inset explains the starting time of the current response curve.

Figure 3 shows the transient current response as the potential was switched from OCP to $0.8 \mathrm{~V}$, using a $90 \%$-duty square waveform oscillating at $0.1 \mathrm{~Hz}$. The attempt to fit the measured current with a single-term exponential function was not satisfactory, confirming the need for a more complex model, such as the twoterm model proposed above. The RSS-minimizing fitting procedure gave an optimized model that agreed well with the experimental data. The exponential term due to the oxidative desorption of $\mathrm{CO}$ contributed to majority of the initial current and decayed relatively quickly with time while the term due to the impedance of the electrochemical system started with a smaller initial value but decayed much more slowly. This general trend $\left(A_{1}>A_{2}\right.$ and $\left.k_{1}>k_{2}\right)$ was seen in the optimized parameters obtained from fitting the transient current response under applied oscillating potentials of different frequencies and duty cycles (Tables S1-S4). The values of $k_{2}$ obtained did not vary significantly across different experimental conditions, which was characteristic of current response due to the electrochemical impedance. Furthermore, as shown in Table S1, the optimized values of $k_{2}$ matched well with the values of $R_{1}=2.873 \Omega, C=4.840 \times 10^{-3} \mathrm{~F}$ and $\frac{1}{R_{1} C}$ $\approx 71.9 \mathrm{~s}^{-1}$ obtained from EIS measurements in a sulfuric acid/FA electrolyte.

Furthermore, the validity of our approach was confirmed by correlating the $\mathrm{CO}_{2}$ formation rate (measured by gas chromatography) with the average rate of electron transfer, obtained by averaging the integrated current during dynamic catalytic reaction conditions over the oscillation period. A linear correlation was found between electron transfer and $\mathrm{CO}_{2}$ productivity with a gradient of $1.7 \pm 0.1 \mathrm{~mol}_{\mathrm{e}}-\mathrm{mol}_{\mathrm{CO}_{2}}^{-1}$ in accordance with the theoretical number of electrons transferred for each molecule of $\mathrm{CO}$ oxidized (=2) within experimental errors (Figure S6a). Good accordance between the number of transferred electrons and the $\mathrm{CO}_{2}$ production rate was found for various static potentials as well (Figure S6b). Those results confirmed that our approach for determining the current due to Faradaic reactions was a viable and experimentally efficient method for quantifying the rate of FA oxidation to $\mathrm{CO}_{2}$.

\subsection{Evaluation of FA dehydration kinetics and CO coverage}

Since the non-Faradaic FA dehydration reaction proceeded during the OCP part of the cycle, the $C O$ coverage could be increased by elongating tOCP and vice versa. With our just established protocol, $\mathrm{CO}$ coverage could be determined by integrating the current response due to the Faradaic $\mathrm{CO}$ oxidative desorption during the whole $t_{0.8 \mathrm{~V}}$.

$$
\begin{aligned}
& \int_{0}^{t_{0.8 \mathrm{~V}}} A_{1} \exp \left(-k_{1} t\right) d t \\
& =\frac{A_{1}}{k_{1}}\left[1-\exp \left(-k_{1} t_{0.8 \mathrm{~V}}\right)\right]
\end{aligned}
$$

For each experiment, $\int_{0}^{\infty} A_{1} \exp \left(-k_{1} t\right) d t$ was also evaluated and verified to be approximately equal (within $0.1 \%$ difference) to $\int_{0}^{t_{0.8 \mathrm{~V}}} A_{1} \exp \left(-k_{1} t\right) d t$, ensuring that practically all $\mathrm{CO}$ had been desorbed from the catalyst surface before the next cycle began. As mentioned earlier, this approach is valid since at $0.8 \mathrm{~V}$, the FA adsorption was reported to be negligible. ${ }^{25} \mathrm{We}$ 
varied the FA dehydration time systematically ( $t_{\mathrm{OCP}}$ ) between $5 \times 10^{-4}$ and $5 \mathrm{~s}$. This was done both by adjusting the duty cycle as well as the frequency of the square waveform in order to avoid artifacts caused by either of those two parameters. For each metal catalyst, the value of $\int_{0}^{t_{0.8 \mathrm{~V}}} A_{1} \exp \left(-k_{1} t\right) d t$ was plotted against the dehydration time. As shown in Figure 4, as the dehydration time increased, the surface coverage reached a maximum value, $\theta_{\max }$. Since differing reports exist on the exact surface fraction that can be covered by $\mathrm{CO}$ with $\theta_{\max }$ values ranging from 0.5 to $0.8,{ }^{28-32}$ the coverage was simply represented as fraction of the maximum coverage, $\theta / \theta_{\max }$, throughout the study:

$$
\frac{\theta}{\theta_{\max }}=\frac{\int_{0}^{t_{0.8 \mathrm{~V}}} A_{1} \exp \left(-k_{1} t\right) d t}{\left(\int_{0}^{t_{0.8 \mathrm{~V}}} A_{1} \exp \left(-k_{1} t\right) d t\right)_{\max }}
$$

Differences between electrodes were mainly assumed to be due to dispersion since the absolute coverages of all three metals are relatively similar. A particularly stark contrast was found for carbon-supported $\mathrm{Pt}$ and $\mathrm{Pt}$ plate electrodes which likely was due to the vast differences in surface areas.

Surprisingly, the times required to achieve $\theta_{\max } / 2$ were $0.19 \mathrm{~s}$ for $\mathrm{Rh} / \mathrm{C}, 0.15 \mathrm{~s}$ for $\mathrm{Pd} / \mathrm{C}, 0.007 \mathrm{~s}$ for $\mathrm{Pt} / \mathrm{C}$ and $0.006 \mathrm{~s}$ for Pt plate. This indicated that the non-Faradaic FA dehydration reaction rates were vastly different for different metals by almost two orders of magnitude but appeared to be insensitive to the structure of the catalyst since $\mathrm{Pt} / \mathrm{C}$ and the $\mathrm{Pt}$ plate electrode exhibited comparable dehydration rates. Since the FA dehydration kinetics follow the trend of $\mathrm{Rh}<\mathrm{Pd} \ll \mathrm{Pt}$ consistent with their observed reactivity, this finding alone appears to be sufficient to rationalize reactivity trends among the three noble metals. ${ }^{33-35}$

\subsection{Coverage-dependent kinetics of $\mathrm{CO}$ oxidative desorption}

After evaluation of the maximum coverage of $\mathrm{CO}$ on the different electrode surfaces, we determined the kinetics for the Faradaic CO oxidative desorption. $k_{1}$ and initial TOF (i.e. at the start of the high potential cycle) were plotted against $\theta / \theta_{\max }$. A power law index, $n$, was used to quantify the variation of $k_{1}$ with $\theta / \theta_{\max }$. As seen in Figure 5a, at full coverage, the reaction rate constants of $\mathrm{Pd} / \mathrm{C}$ and $\mathrm{Rh} / \mathrm{C}$ were comparable and around $100 \mathrm{~s}^{-1}$ while $\mathrm{Pt} / \mathrm{C}$ had roughly three times higher rate constants. We noticed that reaction rate constants were significantly higher at fractional coverage providing a linear correlation between coverage and reaction rate constants in a double-log plot with the power law index $n$ as the slope. Similar $n$ values were determined for the three carbon-supported catalysts $(-0.52,-0.58$, and -0.53 for $\mathrm{Pt} / \mathrm{C}, \mathrm{Pd} / \mathrm{C}$, and $\mathrm{Rh} / \mathrm{C}$, respectively). Pt plate electrodes showed a significantly lower $n$ value of -0.36 indicating the much lower sensitivity of reaction rates to coverage on polycrystalline Pt. It became apparent that the impact of surface $\mathrm{CO}$ coverage on the oxidative desorption activity is almost independent of the metals for nanoparticulate catalysts. However, the morphology of the surface appeared to influence the coveragedependence.

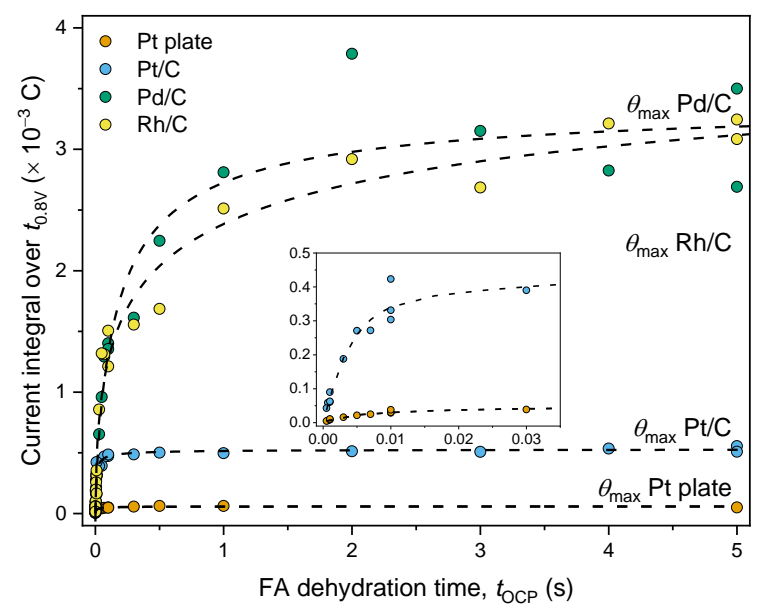

Figure 4. Dependence of integrated current for oxidative CO desorption on the FA dehydration time. The maximum coverage for each electrode is achieved at larger FA dehydration times. The inset shows the enlarged region for $\mathrm{Pt} / \mathrm{C}$ and $\mathrm{Pt}$ plate electrodes.

We noted that the experimentally determined variation of reaction rate constant with coverage implied that the exponential model presented in (7) may not be entirely valid. Therefore, we have quantified the percentage differences between predictions by (7) and a model that allows for coverage-dependent rate constants and shown that they were always $<10 \%$ and thus negligibly small (details in and below Figure S7). Therefore, (7) was nevertheless appropriate for modelling the Faradaic part of the reaction, since most of the oxidative desorption happened in a short time, with a rate constant approximately equal to the value that corresponded to the initial coverage.

Similar trends can be observed in Figure $\mathbf{5 b}$ for the coverage-dependence of the initial turnover frequencies on the different electrodes. Since the TOFs are dependent on the absolute coverage of $\mathrm{CO}$, there is a declining trend as lower relative coverages of $\mathrm{CO}$ are achieved. However, the highest reaction rate constants 

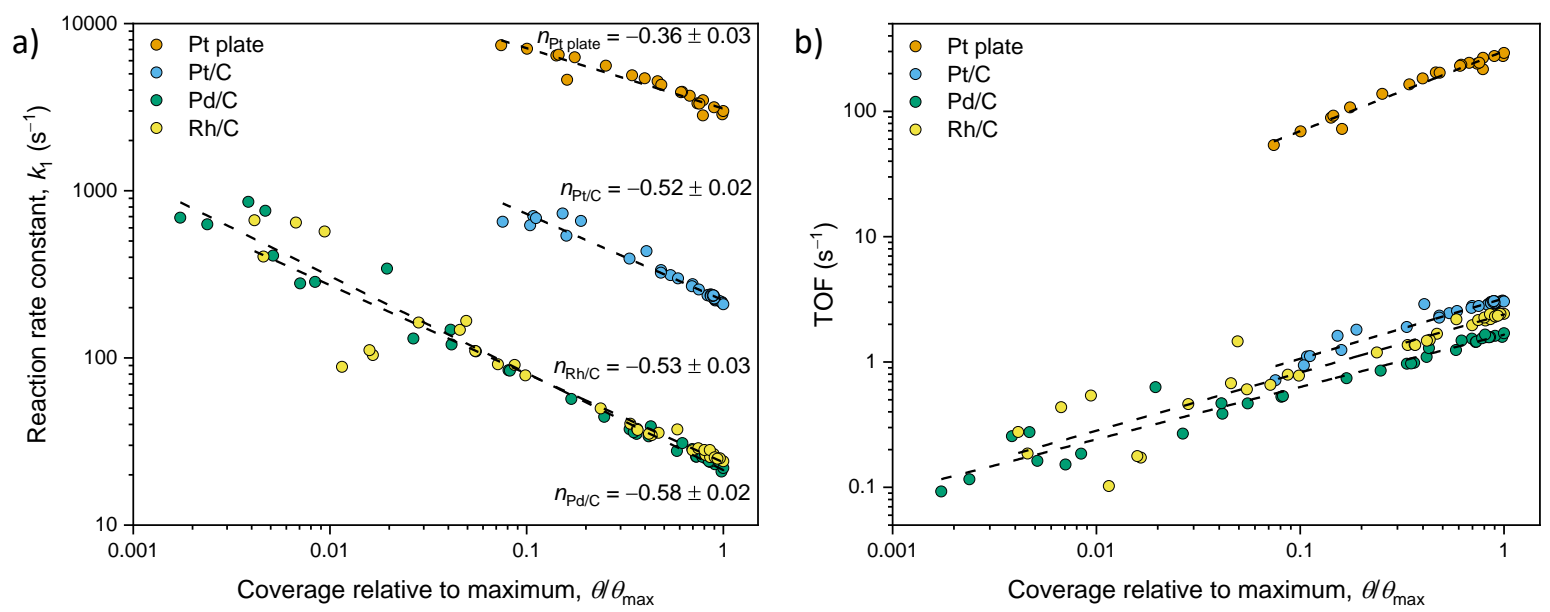

Figure 5. Dependences of reaction rate constant and initial turnover frequency of oxidative desorption of CO on relative surface coverage for different metal catalysts. Values of $n$ for power law " $k_{1}=a \theta^{n "}$ " are shown

are achieved at fractional coverage of the surface with CO. Differences in the $\mathrm{CO}$ oxidative desorption reaction rates are relatively minor compared to the relative rates for FA dehydration as mentioned earlier. This indicates that differences among the three carbonsupported noble metals are almost completely due to differences in the non-Faradaic reaction rates rather than the $\mathrm{CO}$ oxidative desorption. As far as we are aware, not many experimental approaches enable the quantitative kinetic analysis of individual reaction steps of electrocatalytic reactions.

Generally, the flatter Pt plate electrode surface seems to exhibit a much less pronounced coverage dependence and significantly higher reaction rates in comparison to the noble metal nanoparticles. This can in principle be rationalized by the interplay of three effects: a diversity of sites with varying adsorption energies as well as $\mathrm{CO}$ oxidation activities, lateral interactions between adsorbates at higher $\mathrm{CO}$ coverage, as well as different mechanisms dependent on $\theta_{\mathrm{CO}}$ or the type of catalyst. Surface science studies showed that for Pt and Rh surfaces in acidic solutions at positive potentials, lower $\mathrm{CO}$ coverages favored the adsorption of $\mathrm{CO}$ in a bridged fashion. Since we observed that the $\mathrm{CO}$ oxidation reactivity is higher at lower coverage, we could ascribe the highest activity to bridged $\mathrm{CO}$ adsorption sites consistent with previous studies demonstrating that oxidation of bridged $\mathrm{CO}$ on terrace sites occurs at lower over-potentials compared to terminal sites. ${ }^{36}$ This would also help rationalize the vastly enhanced TOF values for the flat $\mathrm{Pt}$ plate electrode which contains more terrace sites suitable for bridged $\mathrm{CO}$ adsorption in contrast to nanoparticles where the curvature favors linearly adsorbed CO. Since the $\mathrm{CO}$ oxidative desorption is believed to follow a Langmuir-Hinshelwood (LH) mechanism on most noble metal surfaces, the co-adsorption of water and $\mathrm{CO}$ is essential. Hence, excessive $\theta_{\mathrm{CO}}$ values prevent the adsorption of co-reactant while the co-adsorption of water was also observed to favor the formation of bridged $\mathrm{CO}$ species further enhancing reactivity. ${ }^{28,37}$

Although contributions of alternative mechanisms like the Eley-Rideal mechanism can be assumed to be negligible under our reaction conditions, ${ }^{38}$ variations for the LH mechanism have been reported before. Island versus random oxidation mechanisms are commonly discussed, the former of which comprises the formation of adsorbed $\mathrm{CO}$ islands which tend to react on the rim while the latter indicates the reaction of a $\mathrm{CO}$ molecule homogeneously located on the electrode surface. Adsorbate islands are a common phenomenon for multimolecular reactions following an LH mechanism since lateral adsorbate interactions often favor spatial segregation of adsorbates. In the case of the $\mathrm{CO}$ oxidative desorption, lateral adsorbateadsorbate interactions are so far rather poorly understood and different effects have been proposed depending on the electrode material. ${ }^{39-40}$ Nonetheless, on the extended surface of the Pt plate electrode, such an island mechanism might indeed play a role and while excessively large islands due to high CO coverage limit the interfacial area between $\mathrm{CO}$ and water adsorbates, a negative $\mathrm{CO}$ reaction order could be easily rationalized. ${ }^{41-43}$ Due to the small particle sizes of carbon-supported electrodes, $\mathrm{CO}$ islands cannot be assumed to exist. The fact that the $\mathrm{Pt}$ plate and nanoparticulate metal electrodes exhibit such distinct TOFs supports the hypothesis that the mechanisms are very different on those surfaces.

\subsection{Optimize dynamic catalytic processes for formic acid oxidation}



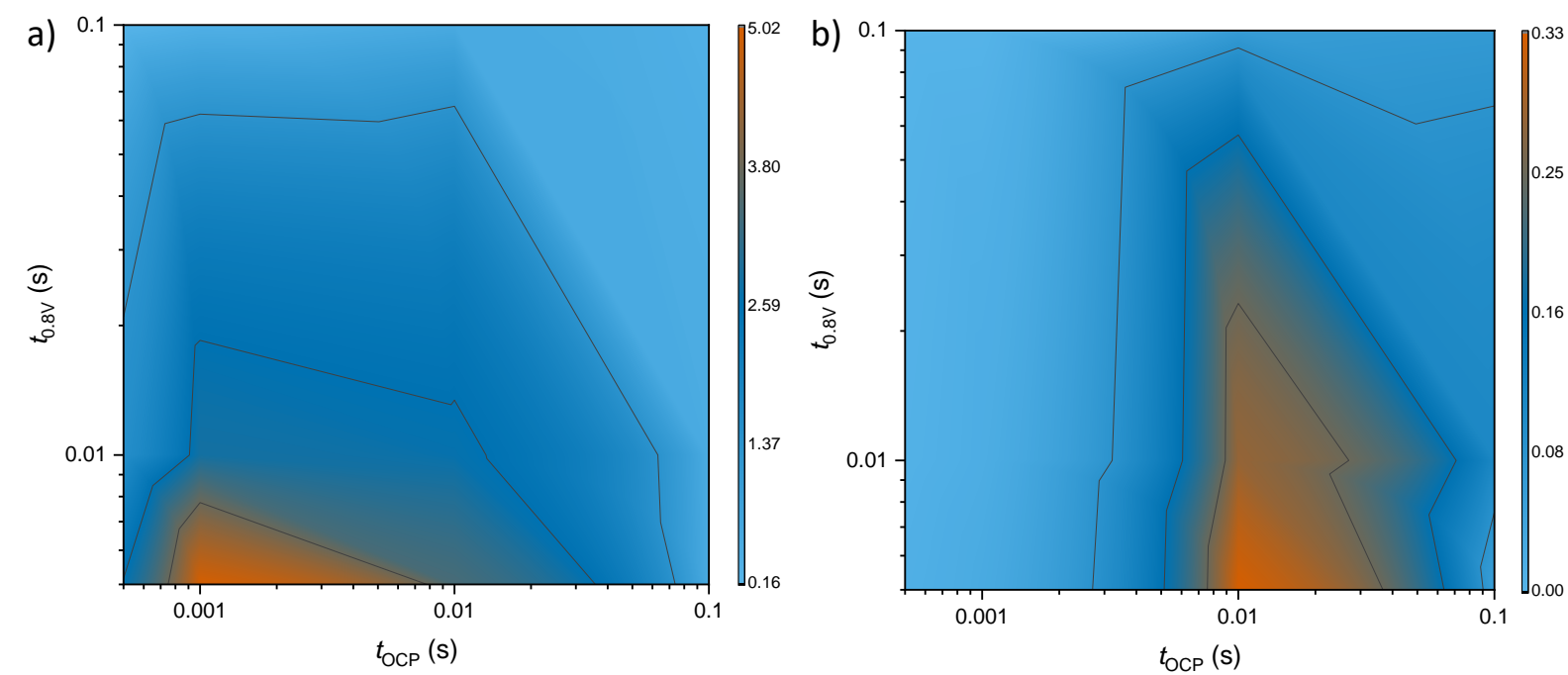

Figure 6. TOF dependence on $t_{0 c P}$ and $t_{0.8 v}$ for a) $\mathbf{P t}$ plate and b) $\mathbf{P d} / \mathbf{C}$. Reactions were conducted with varying frequencies and duty cycles to match the values for $t_{\mathrm{OCP}}$ and $t_{0.8 \mathrm{v}}$. TOF values are given in $\mathrm{s}^{-1}$.

In addition to providing fundamental insights into electrocatalytic reaction kinetics and coveragedependent reaction rates, this tool also help with rationally designing dynamic catalytic processes. Based on the kinetic data, it is relatively straightforward to assume that for different electrodes, dissimilar frequencies and duty cycles might lead to optimized activity. We thus went on to explore the 2dimensional parameter space spanning both $t_{\mathrm{OCP}}$ and $t_{0.8 \mathrm{v}}$ for the $\mathrm{Pd} / \mathrm{C}$ and $\mathrm{Pt}$ plate electrodes (Figure 6), in which TOFs were determined using the integrated current. For the Pt plate electrode, the activity optimum occurs at $t_{\mathrm{OCP}}=0.001 \mathrm{~s}$ while $t_{0.8 \mathrm{~V}}$ should be kept at or below $0.005 \mathrm{~s}$. The ideal dynamic catalytic conditions can thus be achieved at approximately $170 \mathrm{~Hz}$ and a duty cycle of $80 \%$, consistent with previous reports and our own $\mathrm{CO}_{2}$ productivity data (Figure S4). This ideal $t_{\mathrm{OCP}}$ correlates to a $\mathrm{CO}$ coverage of around $50 \%$. Since the time required to achieve a residual $\mathrm{CO}$ coverage of about $0.1 \%$ is $t_{0.8 \mathrm{~V}}=\frac{1}{k_{1}} \times 6=0.002 \mathrm{~s}<0.005 \mathrm{~s}$, conclusions drawn from Figure 6a might overestimate the ideal $t_{0.8 \mathrm{~V}}$ slightly. For $\mathrm{Pd} / \mathrm{C}$ (Figure 6b), ideal $t_{\mathrm{OCP}}$ and $t_{0.8 \mathrm{v}}$ are $0.01 \mathrm{~s}$ and smaller or equal to $0.005 \mathrm{~s}$, respectively. This $t_{\mathrm{OCP}}$ optimum is consistent with an ideal initial CO coverage of roughly $10 \%$ which can be explained by the relatively high susceptibility of the $\mathrm{CO}$ oxidation activity to coverage compared to the Pt plate electrode. The optimum frequency and duty can be assumed to be around $70 \mathrm{~Hz}$ and a duty cycle around $30 \%$. A more distinct optimum $\left(t_{\mathrm{OCP}}=0.01 \mathrm{~s}, t_{0.8 \mathrm{~V}}=\right.$ $0.01 \mathrm{~s}$ ) was found when the FA oxidation reaction was carried out at $270 \mathrm{~K}$ under otherwise identical reaction conditions (Figure S8) with results largely consistent with the reaction at room temperature. This again illustrates that a compromise has been reached between a surface with sufficient fractional coverage without lowering the reaction rate significantly. Electrodes with a larger coverage dependence on the reaction rate exhibit optima at significantly lower coverages, which is usually difficult to accomplish without oscillating potentials. In general, it appears that for reactions with kinetically connected steps as is the case for FA oxidation, correlations between the activity and frequency are not straight-forward and thus kinetics for individual steps must be considered for the design of dynamic catalytic processes.

\section{Conclusions}

In conclusion, we have demonstrated the use of oscillating potentials for the analysis of FA oxidation kinetics. We found that electrodes based on different metals exhibit very different reaction kinetics for the non-Faradaic FA dehydration and the oxidative desorption of $\mathrm{CO}$ rationalizing the performance difference between $\mathrm{Rh}, \mathrm{Pd}$ and Pt. Surprisingly, the main contribution to catalytic activity differences appears to arise from the FA dehydration reaction. Furthermore, we determined coverage-dependent reaction rate constants during the $\mathrm{CO}$ oxidative desorption, with particularly big differences between nanoparticulate and polycrystalline $\mathrm{Pt}$ observed. Although coverage-dependencies of electrocatalytic reactions (e.g. HER, ORR) have been observed and simulated before, we offer one of the first quantitative experimental elucidations. ${ }^{44-46}$ Those results also helped us rationalize and optimize dynamic catalytic processes for formic acid oxidation. We envisage that those insights will be useful to guide the design of dynamic catalytic processes in the future, since the presented approach works in principle for any reaction 
that involves separate non-Faradaic and Faradaic reaction steps.

\section{Acknowledgements}

This research is supported by the NUS Flagship Green Energy Program (R-279-000-553-646, and R-279-000553-731). M. J. H. thanks the SINGA scholarship for supporting his $\mathrm{PhD}$ studies.

\section{References}

1. Adžić, R. R.; Popov, K. I.; Pamić, M. A., Acceleration of electrocatalytic reactions by pulsation of potential: Oxidation of formic acid on $\mathrm{Pt}$ and $\mathrm{Pt} / \mathrm{Pb}_{\mathrm{ads}}$ electrodes. Electrochim. Acta 1978, 23 (11), 11911196.

2. Fedkiw, P. S.; Traynelis, C. L.; Wang, S. R., Pulsed - Potential Oxidation of Methanol. J. Electrochem. Soc. 1988, 135 (10), 2459-2465.

3. Kim, S.; Schoenberg, M. R.; Rappe, A. M., Polarization Dependence of Palladium Deposition on Ferroelectric Lithium Niobate (0001) Surfaces. Phys. Rev. Lett. 2011, 107 (7), 076102.

4. Inoue, Y.; Yoshioka, I.; Sato, K., Polarization effects upon adsorptive and catalytic properties. 1. Carbon monoxide oxidation over palladium deposited on lithium niobate $\left(\mathrm{LiNbO}_{3}\right)$ ferroelectrics. J. Phys. Chem. 1984, 88 (6), 1148-1151.

5. Kakekhani, A.; Ismail-Beigi, S., FerroelectricBased Catalysis: Switchable Surface Chemistry. ACS Catal. 2015, 5 (8), 4537-4545.

6. Neophytides, S. G.; Tsiplakides, D.; Stonehart, P.; Jaksic, M. M.; Vayenas, C. G., Electrochemical enhancement of a catalytic reaction in aqueous solution. Nature 1994, 370 (6484), 45-47.

7 Hülsey, M. J.; Lim, C. W.; Yan, N., Promoting heterogeneous catalysis beyond catalyst design. Chem. Sci. 2020, 11 (6), 1456-1468.

8. Blanco, D. E.; Lee, B.; Modestino, M. A., Optimizing organic electrosynthesis through controlled voltage dosing and artificial intelligence. Proc. Natl. Acad. Sci. U.S.A. 2019, 116 (36), 17683-17689.

9. Ardagh, M. A.; Abdelrahman, O. A.; Dauenhauer, P. J., Principles of Dynamic Heterogeneous Catalysis: Surface Resonance and Turnover Frequency Response. ACS Catal. 2019, 9 (8), 6929-6937.

10. Ardagh, M. A.; Birol, T.; Zhang, Q.; Abdelrahman, O. A.; Dauenhauer, P. J., Catalytic resonance theory: superVolcanoes, catalytic molecular pumps, and oscillatory steady state. Catal. Sci. Technol. 2019, 9 (18), 5058-5076.

11. Ardagh, M. A.; Shetty, M.; Kuznetsov, A.; Zhang, Q.; Christopher, P.; Vlachos, D. G.; Abdelrahman, O. A.; Dauenhauer, P. J., Catalytic resonance theory: parallel reaction pathway control. Chem. Sci. 2020, 11 (13), 3501-3510.

12. Shetty, M.; Walton, A.; Gathmann, S.; Ardagh, M. A.; Gopeesingh, J.; Resasco, J.; Birol, T.;
Zhang, Q.; Tsapatsis, M.; Vlachos, D. G.; Christopher, P.; Frisbie, C. D.; Abdelrahman, O. A.; Dauenhauer, P. J., The Catalytic Mechanics of Dynamic Surfaces: Stimulating Methods for Promoting Catalytic Resonance. ACS Catal., in press.

13. Manish, S.; Matthew, A.; Yutong, P.; Omar, A.; Paul, D., Electric-Field Assisted Modulation of Surface Thermochemistry. ChemRxiv 2020, https://doi.org/10.26434/chemrxiv.12127191.v1.

14. Gopeesingh, J.; Ardagh, M. A.; Shetty, M.; Burke, S. T.; Dauenhauer, P. J.; Abdelrahman, O. A., Resonance-Promoted Formic Acid Oxidation via Dynamic Electrocatalytic Modulation. ACS Catal. 2020, 10 (17), 9932-9942.

15. Chen, Y. X.; Ye, S.; Heinen, M.; Jusys, Z.; Osawa, M.; Behm, R. J., Application of In-situ Attenuated Total Reflection-Fourier Transform Infrared Spectroscopy for the Understanding of Complex Reaction Mechanism and Kinetics: Formic Acid Oxidation on a Pt Film Electrode at Elevated Temperatures. J. Phys. Chem. B 2006, 110 (19), 95349544.

16. Zhang, X.; Choi, I.; Qu, D.; Wang, L.; Lee, C.W. J., Coverage-dependent electro-catalytic activity of $\mathrm{Pt}$ sub-monolayer/Au bi-metallic catalyst toward methanol oxidation. Int. J. Hydrog. Energy 2013, 38 (14), 5665-5670.

17. Park, I.-S.; Chen, D.-J.; Atienza, D. O.; Tong, Y. J., Enhanced CO monolayer electro-oxidation reaction on sulfide-adsorbed $\mathrm{Pt}$ nanoparticles: $\mathrm{A}$ combined electrochemical and in situ ATR-SEIRAS spectroscopic study. Catal.Today 2013, 202, 175-182. 18. Grabow, L. C.; Hvolbæk, B.; Nørskov, J. K., Understanding Trends in Catalytic Activity: The Effect of Adsorbate - Adsorbate Interactions for $\mathrm{CO}$ Oxidation Over Transition Metals. Top. Catal. 2010, 53 (5), 298-310.

19. Borodziński, A.; Bonarowska, M., Relation between Crystallite Size and Dispersion on Supported Metal Catalysts. Langmuir 1997, 13 (21), 5613-5620. 20. Qiu, X.; Zhang, H.; Dai, Y.; Zhang, F.; Wu, P.; Wu, P.; Tang, Y., Sacrificial Template-Based Synthesis of Unified Hollow Porous Palladium Nanospheres for Formic Acid Electro-Oxidation. Catalysts 2015, 5 (2).

21. Zhang, X.; Yin, H.; Wang, J.; Chang, L.; Gao, Y.; Liu, W.; Tang, Z., Shape-dependent electrocatalytic activity of monodispersed palladium nanocrystals toward formic acid oxidation. Nanoscale 2013, 5 (18), 8392-8397.

22. Kucernak, A. R. J.; Fahy, K. F.; Sundaram, V. N. N., Facile synthesis of palladium phosphide electrocatalysts and their activity for the hydrogen oxidation, hydrogen evolutions, oxygen reduction and formic acid oxidation reactions. Catal. Today 2016, 262, 48-56.

23. Sathe, B. R.; Balan, B. K.; Pillai, V. K., Enhanced electrocatalytic performance of interconnected $\mathrm{Rh}$ nano-chains towards formic acid oxidation. Energy Environ. Sci. 2011, 4 (3), 1029-1036. 
24. Xiong, Y.; Dong, J.; Huang, Z.-Q.; Xin, P.; Chen, W.; Wang, Y.; Li, Z.; Jin, Z.; Xing, W.; Zhuang, Z.; Ye, J.; Wei, X.; Cao, R.; Gu, L.; Sun, S.; Zhuang, L.; Chen, X.; Yang, H.; Chen, C.; Peng, Q.; Chang, C.R.; Wang, D.; Li, Y., Single-atom Rh/N-doped carbon electrocatalyst for formic acid oxidation. Nat. Nanotechnol. 2020, 15 (5), 390-397.

25. Brummer, S. B.; Makrides, A. C., Adsorption and Oxidation of Formic Acid on Smooth Platinum Electrodes in Perchloric Acid Solutions. J. Phys. Chem. 1964, 68 (6), 1448-1459.

26. Chen, W.; Kim, J.; Sun, S.; Chen, S., Electrooxidation of formic acid catalyzed by $\mathrm{FePt}$ nanoparticles. Phys. Chem. Chem. Phys. 2006, 8 (23), 2779-2786.

27. Kapusta, S.; Hackerman, N., The Electroreduction of Carbon Dioxide and Formic Acid on Tin and Indium Electrodes. J. Electrochem. Soc. 1983, 130 (3), 607-613.

28. Chang, S.-C.; Weaver, M. J., Coverage- and potential-dependent binding geometries of carbon monoxide at ordered low-index platinum- and rhodium-aqueous interfaces: comparisons with adsorption in corresponding metal-vacuum environments. Surf. Sci. 1990, 238 (1), 142-162.

29. Park, S.; Xie, Y.; Weaver, M. J., Electrocatalytic Pathways on Carbon-Supported Platinum Nanoparticles: Comparison of Particle-SizeDependent Rates of Methanol, Formic Acid, and Formaldehyde Electrooxidation. Langmuir 2002, 18 (15), 5792-5798.

30. Weaver, M. J.; Chang, S. C.; Leung, L. W. H.; Jiang, X.; Rubel, M.; Szklarczyk, M.; Zurawski, D.; Wieckowski, A., Evaluation of absolute saturation coverages of carbon monoxide on ordered low-index platinum and rhodium electrodes. J. Electroanal. Chem. 1992, 327 (1), 247-260.

31. Wang, J.-Y.; Zhang, H.-X.; Jiang, K.; Cai, W.B., From $\mathrm{HCOOH}$ to $\mathrm{CO}$ at Pd Electrodes: A SurfaceEnhanced Infrared Spectroscopy Study. J. Am. Chem. Soc. 2011, 133 (38), 14876-14879.

32. Hahn, F.; Beden, B.; Lamy, C., In situ infrared reflectance spectroscopic study of the adsorption of formic acid at a rhodium electrode. J. Electroanal. Chem. 1986, 204 (1), 315-327.

33. Tang, Y.; Roberts, C. A.; Perkins, R. T.; Wachs, I. E., Revisiting formic acid decomposition on metallic powder catalysts: Exploding the $\mathrm{HCOOH}$ decomposition volcano curve. Surf. Sci. 2016, 650, 103-110.

34. Cuesta, A.; Cabello, G.; Osawa, M.; Gutiérrez, C., Mechanism of the Electrocatalytic Oxidation of Formic Acid on Metals. ACS Catal. 2012, 2 (5), 728-738.

35. Yoo, J. S.; Abild-Pedersen, F.; Nørskov, J. K.; Studt, F., Theoretical Analysis of Transition-Metal Catalysts for Formic Acid Decomposition. ACS Catal. 2014, 4 (4), 1226-1233.

36. McPherson, I. J.; Ash, P. A.; Jones, L.; Varambhia, A.; Jacobs, R. M. J.; Vincent, K. A.,
Electrochemical CO Oxidation at Platinum on Carbon Studied through Analysis of Anomalous in Situ IR Spectra. J. phys. Chem. C 2017, 121 (32), 1717617187.

37. Gilman, S., The Mechanism of Electrochemical Oxidation of Carbon Monoxide and Methanol on Platinum. II. The "Reactant-Pair" Mechanism for Electrochemical Oxidation of Carbon Monoxide and Methanol1. J. Phys. Chem. 1964, 68 (1), 70-80.

38. Urchaga, P.; Baranton, S.; Coutanceau, C.; Jerkiewicz, G., Evidence of an Eley - Rideal Mechanism in the Stripping of a Saturation Layer of Chemisorbed $\mathrm{CO}$ on Platinum Nanoparticles. Langmuir 2012, 28 (36), 13094-13104.

39. Rodriguez, P.; Garcia-Araez, N.; Koper, M. T. M., Self-promotion mechanism for CO electrooxidation on gold. Phys. Chem. Chem. Phys. 2010, 12 (32), 9373-9380.

40. Gómez-Marín, A. M.; Hernández-Ortiz, J. P., Langmuir - Hinshelwood Mechanism Including Lateral Interactions and Species Diffusion for $\mathrm{CO}$ Electro-Oxidation on Metallic Surfaces. J. Phys. Chem. C 2014, 118 (5), 2475-2486.

41. Veser, G.; Esch, F.; Imbihl, R., Regular and irregular spatial patterns in the catalytic reduction of NO with NH3 on $\mathrm{Pt}(100)$. Catal. Lett. 1992, 13 (4), 371-382.

42. Zhdanov, V. P., Pattern Formation in Catalytic Reactions Due to Lateral Adsorbate-Adsorbate Interactions. Langmuir 2001, 17 (5), 1793-1799.

43. Barroo, C.; Wang, Z.-J.; Schlögl, R.; Willinger, M.-G., Imaging the dynamics of catalysed surface reactions by in situ scanning electron microscopy. Nat. Catal. 2020, 3 (1), 30-39.

44. Shinagawa, T.; Garcia-Esparza, A. T.; Takanabe, K., Insight on Tafel slopes from a microkinetic analysis of aqueous electrocatalysis for energy conversion. Sci. Rep. 2015, 5 (1), 13801.

45. Fu, W.; Shu, S.; Li, J.; Shi, X.; Lv, X.; Huang, Y.-X.; Dong, F.; Jiang, G., Identifying the ratedetermining step of the electrocatalytic hydrodechlorination reaction on palladium nanoparticles. Nanoscale 2019, 11 (34), 15892-15899. 46. Fang, Y.-H.; Liu, Z.-P., Tafel Kinetics of Electrocatalytic Reactions: From Experiment to FirstPrinciples. ACS Catal. 2014, 4 (12), 4364-4376. 